\title{
Time Scale Decomposition in Complex Reaction Systems: A Graph Theoretic Analysis
}

\author{
Udit Gupta, Seongmin Heo, Aditya Bhan*, Prodromos Daoutidis* \\ Department of Chemical Engineering and Materials Science, University of Minnesota, MN 55455 USA
}

\begin{abstract}
The formulation of a kinetic model for a complex reaction network typically yields reaction rates which vary over orders of magnitude. This results in time scale separation that makes the model inherently stiff. In this work, a graph-theoretic framework is developed for time scale decomposition of complex reaction networks to separate the slow and fast time scales, and to identify pseudo-species that evolve only in the slow time scale. The reaction network is represented using a directed bi-partite graph and cycles that correspond to closed walks are used to identify interactions between species participating in fast/equilibrated reactions. Subsequently, an algorithm which connects the cycles to form the pseudo-species is utilized to eliminate the fast rate terms. These pseudo-species are used to formulate reduced, nonstiff kinetic models of the reaction system. Two reaction systems are considered to show the efficacy of this framework in the context of thermochemical and biochemical processing.
\end{abstract}

Keywords: Graph theory; Model reduction; Bi-partite graph; Lumping

\section{INTRODUCTION}

Complex reaction networks are present in numerous chemical and biochemical systems, such as combustion, pyrolysis, nanoparticle synthesis, catalytic conversion of hydrocarbons, and cell metabolism. These reaction networks are of particular recent interest because of the emergence of new feedstocks and chemistries, e.g. for biomass and methane processing. Microkinetic modeling is an essential step towards rigorous design, optimization, and control of these reaction systems; however, the development of microkinetic models, with the underlying parameter estimation problem, is computationally challenging, with two key challenges being model stiffness and size. Stiffness arises from the difference in the order of magnitude of reaction rate constants, while the large model size is due to the large number of species and reactions typically present in such networks. Although there exist numerical methods for simulation of large scale, stiff models, the use of such models in optimization-based tasks (e.g. parameter estimation, control) results in ill-conditioning of the corresponding optimization task. Model reduction methods for kinetic simplification involving lumping, sensitivity analysis, and time-scale analysis are generally used to address these challenges (Okino and Mavrovouniotis, 1998).

${ }^{*}$ Corresponding author

Email addresses: abhan@umn.edu (Aditya Bhan), daout0010umn.edu (Prodromos Daoutidis) 
Model reduction methods based on time-scale analysis include numerical approaches like the computational singular perturbation method (Lam and Goussis, 1989, 1994; Massias et al., 1999) where the eigenvalues of the Jacobian of the kinetic system of differential equations are used to identify the slow invariant manifold (Fenichel, 1979); the intrinsic low-dimensional manifold method (Maas and Pope, 1992) where an eigenvalue-eigenvector decomposition of the Jacobian matrix is performed with the assumption that the fast subspace vanishes quickly (Pope, 1997; Yang and Pope, 1998); geometric-based analysis (Fraser, 1988; Roussel and Fraser, 1991) where a comprehensive investigation of the features of trajectories in the concentration phase space starting from many different initial conditions is used; and analytical, projection-based methods (Vora and Daoutidis, 2001; Gerdtzen et al., 2004; Adomaitis, 2016; Remmers et al., 2015; Lee and Othmer, 2010; Prescott and Papachristodoulou, 2014). All of these methods, however, require considerable computational effort in practical applications to complex, large scale systems (Lebiedz, 2004) and have been mostly applied to homogeneous reaction systems.

Alternatively, mechanism reduction methods based on reaction rate evaluation (Lu and Law, 2006; Lu et al., 2009; Pepiot-Desjardins and Pitsch, 2008; Susnow et al., 1997) allow elimination of unimportant species and reactions from the reaction network, thereby, reducing the computational complexity of the system. The reaction rate evaluation is possible when the system has well-defined kinetics like in the case of gas-phase chemistry, due to the existence of a vast kinetic database for gas-phase chemical reactions (NIST Chemical Kinetics Database). For systems where there is significant uncertainty in the kinetic parameters, eliminating unimportant species and reactions based on an approximate set of kinetic constants may lead to erroneous results.

In this research, a graph-theoretic framework is proposed for generation of non-stiff reduced models of isothermal reaction systems with fast and slow reactions. A directed bi-partite graph is used to represent the reaction network and the reactions are characterized as fast or slow using a kinetic threshold and an equilibrium tolerance. Cycles that correspond to closed walks are then used to identify interactions between species participating in fast/equilibrated reactions. Subsequently, an algorithm which connects these cycles to generate pseudo-species that evolve in the slow time scale alone is presented. The result is an automated, generic procedure for generating non-stiff reduced models in terms of these pseudo-species, while enforcing typical quasi-equilibrium or complete conversion constraints for fast reactions. The efficacy of the developed framework is illustrated through its application on two chemical reaction systems: 1-butene cracking and carbon metabolism in erythrocytes.

\section{METHODOLOGY}

Consider a reaction network of a homogeneous, isothermal system with $n$ chemical species $(S)$ and $m$ reactions $(R)$, with $\alpha_{i j}$ and $\beta_{i j}$ the stoichiometric coefficients of the reactants and products, respectively, and $k_{i}$ the kinetic constant for reaction $R_{i}$ :

$$
\sum_{j=1}^{n} \alpha_{i j} S_{j} \stackrel{k_{i}}{\longrightarrow} \sum_{j=1}^{n} \beta_{i j} S_{j}, \quad i=1, \ldots, m
$$


Let $C_{j}$ be the concentration of species $S_{j}$ and $\mathrm{C}=\left(C_{1}, C_{2}, \ldots, C_{n}\right)^{T}$ be the vector of concentrations. The reaction rate $r_{i}$ is generally expressed as a product of a reaction rate constant, $k_{i}$, and a nonlinear function of concentrations, $f_{i}(\mathrm{C})$ :

$$
r_{i}(\mathrm{C})=k_{i} f_{i}(\mathrm{C})
$$

In the case of reversible reactions, the forward and reverse reactions are represented separately in Eq. 1. A kinetic model of a batch (fixed volume) system, derived from the mass balances for the species, results in a set of ordinary differential equations (ODEs) that gives the time evolution of the concentrations, $C_{j}, j=1, \ldots, n$ :

$$
\frac{d C_{j}}{d t}=\sum_{i}\left(\beta_{i j}-\alpha_{i j}\right) \times r_{i}(\mathrm{C})
$$

In a plug flow reactor, these ODEs are reformulated with respect to reactor volume $(V)$. The spatial evolution of the molar flow rates, $F_{j}, j=1, \ldots, n$, at steady state is then given by:

$$
\frac{d F_{j}}{d V}=\sum_{i}\left(\beta_{i j}-\alpha_{i j}\right) \times r_{i}(\mathrm{C})
$$

In a heterogeneous (gas-solid) system, the quasi-steady-state assumption (QSSA) for surface intermediates is typically employed (Bowen et al., 1963) involving adsorption/desorption reactions. Let $Q$ be the subset of $S=\left\{S_{1}, S_{2}, \ldots, S_{n}\right\}$ containing the surface intermediates. Then the QSSA assumption applied on these species results in the following algebraic equations

$$
\sum_{i}\left(\beta_{i j}-\alpha_{i j}\right) \times r_{i}(\mathrm{C})=0 \quad \forall j \in Q
$$

Together with the differential equations for the gas-phase species, the kinetic model in this case is a differential-algebraic equation (DAE) system.

The framework developed in the present work is applicable to all types of reaction systems discussed above. Model stiffness can result from large reaction rate constants in the case of irreversible reactions or high forward/reverse reaction rates in the case of reversible reactions. A systematic framework is developed for identifying such fast/equilibrated reactions and generating pseudo-species evolving in a slow time scale, while enforcing quasi-equilibrium or complete conversion constraints. The steps involved are: (1) graph representation for the reaction network, (2) identification of fast/equilibrated reactions, (3) identification of fast sub-graphs, (4) identification of cycles, and (5) generation of pseudo-species. Each of these steps is discussed in detail below.

\subsection{Graph representation for the reaction network}

A directed bi-partite graph $\mathcal{G}_{B}=(S, R, E)$ with two disjoint sets of vertices, one including species $(S)$ and the other including reactions $(R)$ (Mincheva and Roussel, 2007; Holme et al., $2003)$, and the set of directed links $(E)$ - ordered pairs of one node in $S$ and one node in $R$ - is used to represent the reaction network. A species is identified as a reactant/product based on the direction of the edge. An edge directed from the species set to the reaction set 
implies that the species is a reactant, while an edge directed from the reaction set to the species set implies that the species is a product. An example reaction scheme along with its graph representation is shown in Figure 1. The bi-partite graph allows representation of both bimolecular and unimolecular reactions as opposed to other graphical representations (e.g., a digraph with nodes denoting species and edges denoting reactions which can only capture unimolecular reactions (Domijan and Kirkilionis, 2008)).

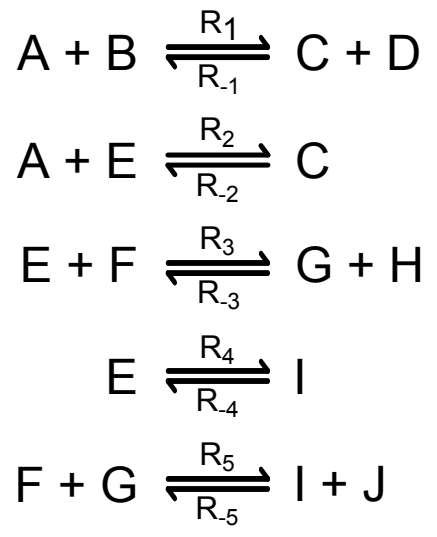

(a)

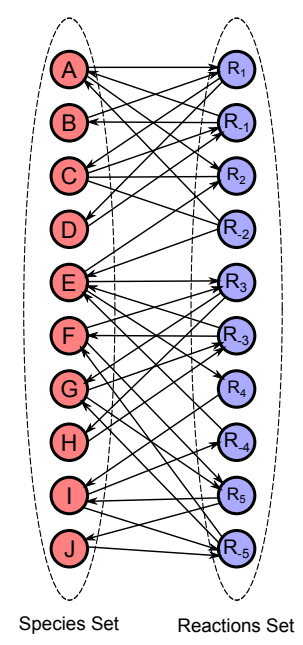

(b)

Figure 1: (a) Reaction scheme with species represented as letters and $R_{1} / R_{-1}, R_{2} / R_{-2}, R_{3} / R_{-3}, R_{4} / R_{-4}$, and $R_{5} / R_{-5}$ representing reactions and (b) its directed bi-partite representation.

\subsection{Identification of fast/equilibrated reactions}

In the next step, the reactions are classified as fast and slow using a kinetic threshold $\left(k^{\text {min }}\right)$ and an equilibrium tolerance $(\delta)$. For irreversible reactions, a kinetic threshold value, $k^{\text {min }}$ (where $k^{\text {min }}$ has dimensions of inverse time) is assumed and any reaction with a pseudo-firstorder rate constant above this threshold is considered to be fast. Note that for a bimolecular reaction, the reaction rate constant can be normalized to get the units of inverse time by using the concentration of one of the reactants present in excess or in case of biochemical systems, using enzyme activity, enzyme concentration, or the average concentration of some of the species (Heinrich et al., 1978).

Reversible reactions that equilibrate over a short initial time (or space time) have fast forward and reverse rates. These reactions can be similarly identified based on the kinetic threshold, however, a reaction may not satisfy the equilibrium condition if the concentrations of species in the reaction vary over several orders of magnitude. Hence, fast reversible reactions that equilibrate are identified by defining a term, the equilibrium index, which captures the ratio of the forward reaction rate and the reverse reaction rate:

$$
\text { Equilibrium Index }=\frac{\text { Forward reaction rate }}{\text { Reverse reaction rate }}=\frac{k_{\text {forward }} \times f_{\text {forward }}(\mathrm{C})}{k_{\text {reverse }} \times f_{\text {reverse }}(\mathrm{C})}
$$

An equilibrated reaction will have an equilibrium index value of unity. An equilibrium tolerance $\delta$, will be used to characterize reactions that can be assumed equilibrated. To this 
end, the forward and reverse reaction rates of a reversible reaction are calculated through a forward simulation of the ODEs (using best available estimates of kinetic parameters if these are not known exactly). The equilibrium index is calculated for each reversible reaction over the whole spatial/temporal region of interest; reactions with equilibrium index values always lying within the tolerance $\delta$, are considered equilibrated:

$$
\left|1-\frac{k_{\text {forward }} \times f_{\text {forward }}(\mathrm{C})}{k_{\text {reverse }} \times f_{\text {reverse }}(\mathrm{C})}\right| \leq \delta
$$

Note that in the case of reversible reaction, we use the same number for both the forward and reverse reactions, but with a negative sign for the latter as shown in figure 1a.

Algorithm 1 below describes the steps for identifying the fast/equilibrated reactions. Two datastructures, FastReactionList $\left(E_{\text {fast }}\right)$ and SlowReactionList $\left(E_{\text {slow }}\right)$ are generated for the fast and slow reaction edges respectively, and two datastructures, ReactantMap $\left(R M_{j}\right)$ and ProductMap $\left(P M_{j}\right)$. ReactantMap contains reactions in which species $S_{j}$ participates as a reactant whereas ProductMap contains reactions in which species $S_{j}$ participates as a product.

\begin{tabular}{l} 
Algorithm 1 Reaction Identification $\left(\mathcal{G}_{\mathcal{B}}(S, R\right.$ \\
\hline 1: Forward simulation $\left(C_{0}, k\right)$ \\
2: for $\mathrm{i}=1: \operatorname{size}(E)$ do \\
3: if $E_{i} \in \mathcal{I} \mathcal{R} \mathcal{S}$ then \\
4: $\quad$ checkThresholdCriterion $\left(E_{i}\right)$ \\
5: else if $E_{i} \in \mathcal{R S}$ then \\
6: checkEquilibriumIndexCriterion $\left(E_{i}\right)$ \\
7: $\quad$ end if
\end{tabular}

8: $\quad$ if fastReaction $\left(E_{i}\right)$ then

9: $\quad$ Put $E_{i}$ in $E_{\text {fast }}$

10: $\quad$ for $\mathrm{j}=1: \operatorname{size}\left(\mathcal{N}_{R}\right)$ do

11: $\quad$ Put $E_{i}$ in $R M_{j}$ for $S_{j}$

12: $\quad$ end for

13: $\quad$ for $\mathrm{j}=1: \operatorname{size}\left(\mathcal{N}_{P}\right)$ do

14: $\quad$ Put $E_{i}$ in $P M_{j}$ for $S_{j}$

15: $\quad$ end for

16: else

17: $\quad$ Put $E_{i}$ in $E_{\text {slow }}$

18: end if

19: end for $\triangleright$ Perform a forward simulation of the ODEs to calculate the equilibrium index of reversible reactions.

$\triangleright$ Check the type of the corresponding reaction (reversible or irreversible) and also whether the reaction satisifies the identification criterion for fast reactions. The routines checkThresholdCriterion $\left(E_{i}\right)$ and checkEquilibriumIndexCriterion $\left(E_{i}\right)$ examine irreversible and reversible reactions respectively.

$\triangleright$ The routine fastReaction $\left(E_{i}\right)$ checks if edge $E_{i}$ is fast and two datastructures, FastReactionList $\left(E_{\text {fast }}\right)$ and SlowReactionList $\left(E_{\text {slow }}\right)$ are generated for the fast and slow reaction edges respectively. ReactantMap $\left(R M_{j}\right)$ contains reactions in which species $S_{j}$ participates as a reactant and ProductMap $\left(P M_{j}\right)$ contains reactions in which species $S_{j}$ participates as a product. 


\subsection{Identification of fast sub-graphs}

The sub-graphs in $\mathcal{G}_{\mathcal{B}}(S, R, E)$ which contain only fast reaction edges and are connected with the remaining reaction network through slow reactions only are identified in this next step. Figure 2a shows the same reaction scheme as in Figure 1a, where some of reactions $\left(R_{1} / R_{-1}\right.$, $\left.R_{3} / R_{-3}, R_{4} / R_{-4}\right)$ are considered fast and the corresponding sub-graphs are explicitly identified. The fast reactions are shown with red arrows while the slow reactions are shown using black arrows. It can be seen that only slow reactions (black arrows) pass through an enclosed dashed boundary, illustrating that sub-graphs comprising only fast reactions interact with the remaining reaction network through slow reactions only.

$$
\begin{array}{r}
A+B \underset{R_{-1}}{\stackrel{R_{1}}{\rightleftharpoons}} C+D \\
A+E \stackrel{R_{2}}{\underset{R_{-2}}{\rightleftharpoons}} C \\
E+F \underset{R_{-3}}{\stackrel{R_{3}}{\rightleftharpoons}} G+H \\
\quad E \stackrel{R_{4}}{\stackrel{R_{-4}}{\rightleftharpoons}} I \\
F+G \underset{R_{-5}}{\stackrel{R_{5}}{\rightleftharpoons}} I+J
\end{array}
$$

(a)

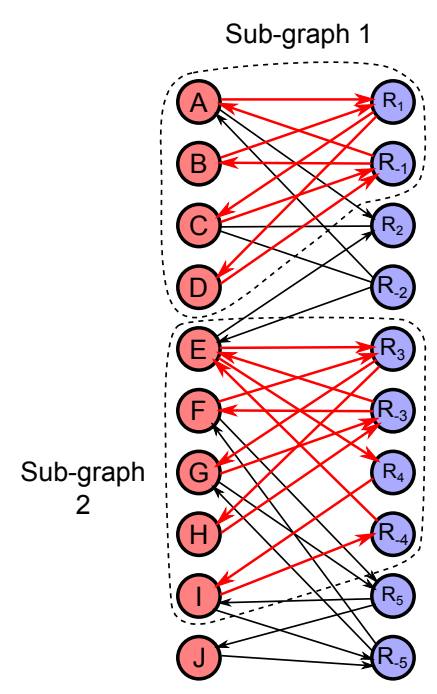

(b)

Figure 2: (a) Reaction scheme with the $R_{1} / R_{-1}, R_{3} / R_{-3}, R_{4} / R_{-4}$ reactions considered fast and (b) the directed bi-partite representation illustrating the identified fast sub-graphs.

In a general reaction network, these sub-graphs can be identified using a breadth-first search (BFS) graph traversal algorithm (Cormen et al., 2001). Algorithm 2 below describes the steps followed in identifying the fast sub-graphs. In the general case, the procedure runs over all species $S$ and checks if species $S_{j}$ participates in a fast reaction. If a species participates in a fast reaction, it is added to a SpeciesQueue and in a sub-graph, $\mathcal{S G}$. Using species $S_{j}$, the procedure runs over the reactions in the ReactantMap, $R M$ and the reactions in the ProductMap, $P M$, finding the product and reactant species, respectively, of the reactions that $S_{j}$ participates in. These species are then added to the SpeciesQueue and to the same sub-graph as species $S_{j}$. If a reaction is bimolecular, the co-reactant is also found and added to the SpeciesQueue and the same sub-graph. Further, the species $S_{j}$ is removed from the SpeciesQueue and the next species in the queue is selected, and the procedure is repeated. The procedure terminates when the SpeciesQueue is empty resulting in a list of all fast subgraphs in the reaction network. If a species does not participate in any fast reaction, the species is added to the true slow species datastructure, $\mathcal{T S}$. 


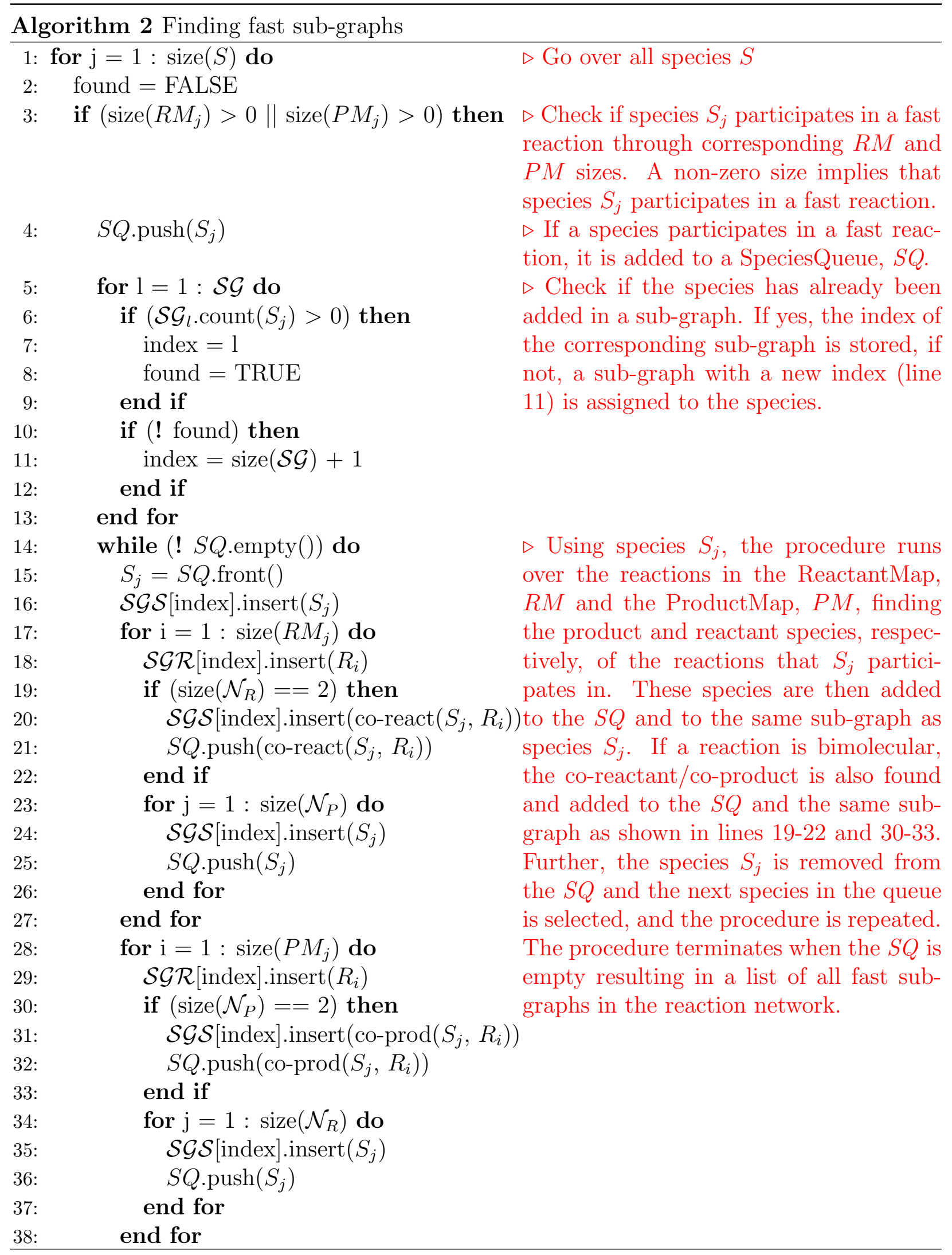




$\begin{array}{lc}\text { 39: } & S Q \cdot \operatorname{pop}() \\ \text { 40: } & \text { end while } \\ \text { 41: } & \text { else } \\ \text { 42: } & \mathcal{T} \mathcal{S} \cdot \operatorname{insert}\left(S_{j}\right) \\ \text { 43: } & \text { end if } \\ \text { 44: } & \text { end for }\end{array}$

$\triangleright$ If a species does not participate in any fast reaction, the species is added to the true slow species datastructure, $\mathcal{T S}$.

\subsection{Identification of cycles}

The interactions between species participating in fast reactions within each sub-graph are identified in this step. Specifically, cycles are identified that correspond to closed walks over the fast edges in the graph. Figure 3 shows sub-graph 1 identified in Figure 2 with reaction $R_{1} / R_{-1}$ considered as fast. The procedure for identifying cycles involves starting at a species node, e.g. node $\mathrm{A}$ or node $\mathrm{B}$, traversing in the direction of the arrow to the reaction node, $\mathrm{R}_{1}$ (corresponding to the fast reaction), traversing to one of the products of the reaction, e.g. node $\mathrm{C}$ or node $\mathrm{D}$, traversing to the node for the corresponding reverse reaction, $\mathrm{R}_{-1}$, and finally, traversing back to the starting species node, to complete the cycle. The cycles identified for this fast bimolecular reaction are shown in Figure 3.

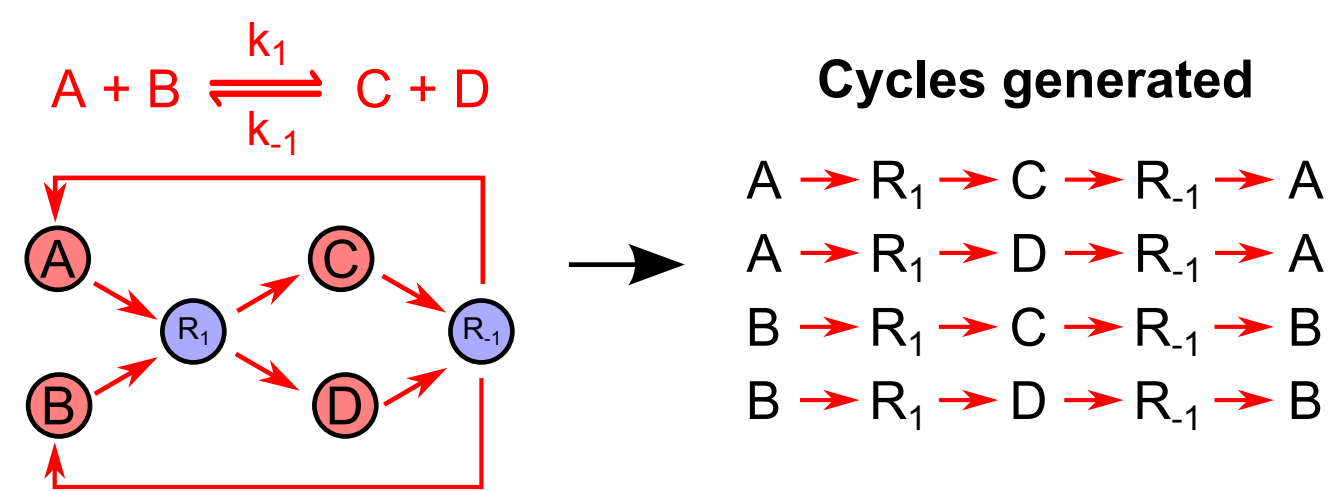

Figure 3: Cycles generated for a fast bi-molecular reaction.

For a general sub-graph involving more than one reaction, the backtrack algorithm (Tarjan, 1973) is used to generate the cycles for all the species and fast reactions. Algorithm 3 below describes the steps followed in generating cycles for reactions in a fast sub-graph. The procedure runs over all sub-graphs, $\mathcal{S G}$. Within each sub-graph, the algorithm runs over each species, present in the sub-graph, stored in datastructure $\mathcal{S G S}$. The species $S_{j}$ is used as a starting node to generate the cycle for the reactions that it participates in. The algorithm then goes over all the reactions that use species $S_{j}$ as a reactant, finding the second node in the cycle. $w_{i}$ stores the stoichiometric coefficient of the reactant $R_{i}$ using the routine stoichiometry $\left(S_{j}, R_{i}\right)$ which requires the species and reaction as inputs. Next, the algorithm goes over the products of the reaction $R_{i}$, finding the third node of the cycle. The fourth node corresponding to the reverse reaction is found using the definition for the routine reverse $\operatorname{Rxn}\left(R_{i}\right) . w_{-i}$ stores the stoichiometric coefficient of the reactant $R_{-i}$ 
using the routine stoichiometry $\left(S_{t}, R_{-i}\right)$. The information about stoichiometric coefficients and species pair in a cycle for a reaction is stored in datastructures $\mathcal{N P}$ and $\mathcal{N P \mathcal { I }}$. The procedure ends when all species within a sub-graph and all sub-graphs are processed.

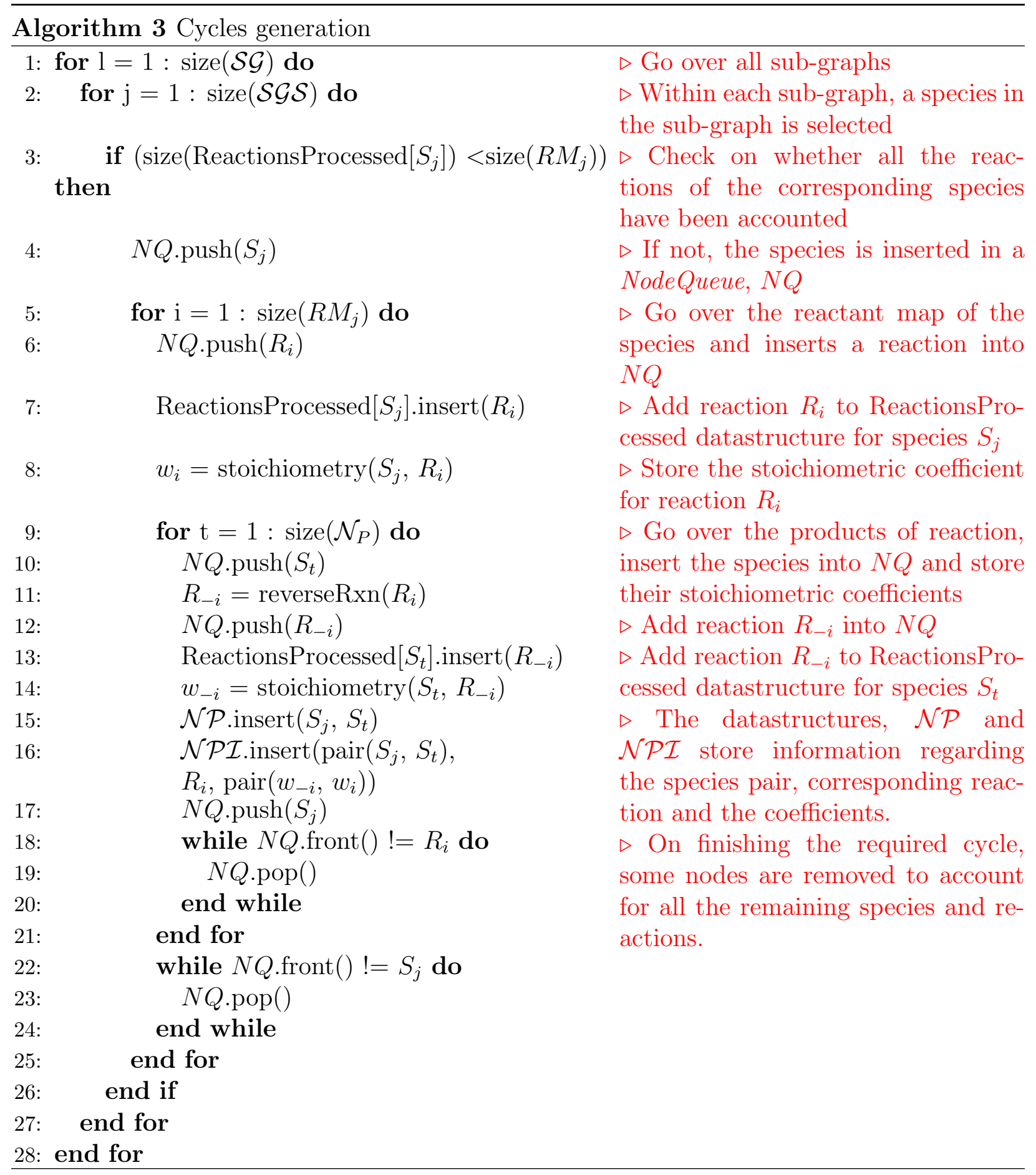

\subsection{Identification of pseudo-species using the cycles}

From each cycle, a pseudo-species (the sum of the two species involved in the cycle) can be readily identified such that the contributions of the fast reaction rates cancel out. For exam- 
ple, for the first cycle, $\mathrm{A} \rightarrow \mathrm{R}_{1} \rightarrow \mathrm{C} \rightarrow \mathrm{R}_{-1} \rightarrow \mathrm{A}$, as shown in Figure 3, the corresponding pseudo-species is $(A+C)$. Eq. 8 and Eq. 9 show the mass balances for species $\mathrm{A}$ and $\mathrm{C}$ respectively. Both equations contain the fast reaction rate terms corresponding to the fast bimolecular reaction $\left(k_{-1} f_{-1}(\mathrm{C})\right.$ and $\left.k_{1} f_{1}(\mathrm{C})\right)$ and slow reaction rate terms corresponding to the slow reactions, $\sum S . T_{1}$ and $\sum S . T_{3}$ for species $\mathrm{A}$ and $\mathrm{C}$ respectively. Eq. 10 shows the mass balance for the pseudo-species $(A+C)$, where only the slow reaction rate terms are present with the fast reaction rate terms being eliminated. Similarly, the other pseudospecies corresponding to the other identified cycles are $A+D, B+C$, and $B+D$. Note that one of these species is linearly dependent on the remaining three species. Therefore, the fourth species $(B+D)$ should be removed from the set of pseudo-species to avoid such a redundancy. The choice of this species is arbitrary implying that the set of pseudo-species generated is not unique.

\section{Original Model}

$\frac{d C_{A}}{d t}=k_{-1} f_{-1}(\mathrm{C})-k_{1} f_{1}(\mathrm{C})+\sum S . T_{1}$
$\frac{d C_{C}}{d t}=-k_{-1} f_{-1}(\mathrm{C})+k_{1} f_{1}(\mathrm{C})+\sum S . T_{3}$

Model in terms of pseudo-species

$$
\frac{d\left[C_{A}+C_{C}\right]}{d t}=\sum S . T_{1}+\sum S . T_{3}
$$

Since each cycle can be considered as a species pair, the occurrences of a species pair in all fast reactions are stored, to be used later for generating pseudo-species.

Remark: The generation of pseudo-species following the above procedure corresponds to a particular choice of coordinate change such that the corresponding coefficient matrix belongs to the left null space of the stoichiometric matrix of the fast reactions. For the above subgraph the fast reaction stoichiometric matrix is $\left[\begin{array}{c}-1 \\ -1 \\ 1 \\ 1\end{array}\right]$ and the choice of pseudo-species

corresponds to a coordinate matrix $\left[\begin{array}{llll}1 & 0 & 0 & 1 \\ 0 & 1 & 1 & 0 \\ 0 & 1 & 0 & 1\end{array}\right]$ with $\left[\begin{array}{llll}1 & 0 & 0 & 1 \\ 0 & 1 & 1 & 0 \\ 0 & 1 & 0 & 1\end{array}\right] \times\left[\begin{array}{c}-1 \\ -1 \\ 1 \\ 1\end{array}\right]=0$. In this sense, the proposed procedure implements in a graph-theoretic setting the projection-based approach in Vora and Daoutidis (2001), Gerdtzen et al. (2004).

\subsubsection{Species participating in multiple reactions within a sub-graph}

In a general sub-graph, a species can participate in multiple fast reactions. Figure 4a shows the third and the fourth reactions from Figure 1 considered as fast along with the directed bi-partite representation of the corresponding sub-graph from Figure $2 b$. 


$$
\begin{gathered}
E+F \underset{R_{-3}}{\stackrel{R_{3}}{\rightleftharpoons}} \mathrm{G}+\mathrm{H} \\
E \stackrel{\mathrm{R}_{4}}{\underset{R_{-4}}{\rightleftharpoons}} \text { I }
\end{gathered}
$$

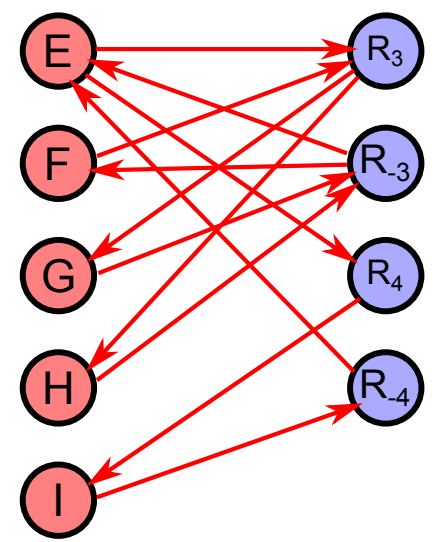

(b)

Figure 4: (a) Reaction scheme with $R_{3} / R_{-3}$ and $R_{4} / R_{-4}$ reactions considered fast and (b) the directed bi-partite representation of the corresponding sub-graph.

The species $\mathrm{E}$ participates in both reactions which contribute fast rate terms $\left(k_{-3} f_{-3}(\mathrm{C})\right.$, $k_{3} f_{3}(\mathrm{C}), k_{-4} f_{-4}(\mathrm{C})$, and $\left.k_{4} f_{4}(\mathrm{C})\right)$ shown in Eq. 11. Therefore, to eliminate all fast reaction rate terms from the corresponding mass balance, the cycles identified for each reaction need to be combined. Considering the cycles from each reaction, $\mathrm{E} \rightarrow \mathrm{R}_{3} \rightarrow \mathrm{G} \rightarrow \mathrm{R}_{-3} \rightarrow \mathrm{E}$ for the first reaction and $\mathrm{E} \rightarrow \mathrm{R}_{4} \rightarrow \mathrm{I} \rightarrow \mathrm{R}_{-4} \rightarrow \mathrm{E}$ for the second reaction, the pseudo-species that will be invariant in the fast time scale is $E+G+I$. The mass balance for this pseudospecies is shown in Eq. 14 and indeed involves only slow reaction terms. Figure 5 illustrates the combination of the two cycles which generates the above pseudo-species. Similarly, this procedure can be applied to the second cycle for species $E$ in reaction $R_{3}$ to generate the pseudo-species $E+H+I$, as well as for the other species in the sub-network.

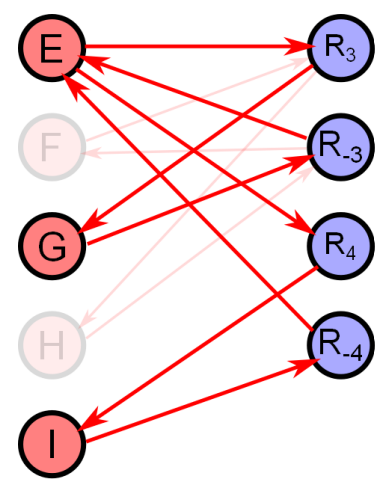

$$
E+G+I
$$

Figure 5: Cycles correponding to fast reactions of species E used to generate the pseudo-species 


\title{
Original Model
}

$$
\begin{aligned}
\frac{d C_{E}}{d t} & =k_{-3} f_{-3}(\mathrm{C})-k_{3} f_{3}(\mathrm{C})+k_{-4} f_{-4}(\mathrm{C})-k_{4} f_{4}(\mathrm{C})+\sum S . T_{5} \\
\frac{d C_{G}}{d t} & =-k_{-3} f_{-3}(\mathrm{C})+k_{3} f_{3}(\mathrm{C})+\sum S . T_{7} \\
\frac{d C_{I}}{d t} & =-k_{-4} f_{-4}(\mathrm{C})+k_{4} f_{4}(\mathrm{C})+\sum S . T_{9}
\end{aligned}
$$

\section{Model in terms of pseudo-species}

$$
\frac{d\left[C_{E}+C_{G}+C_{I}\right]}{d t}=\sum S . T_{5}+\sum S . T_{7}+\sum S . T_{9}
$$

\begin{abstract}
Algorithm 4 shown below describes the steps followed in the pseudo-species generation procedure by combining the cycles identified in section 2.4. The species-pairs are sorted based on their occurrences in datastructure $\mathcal{N} \mathcal{P}$. The procedure starts from the most frequently occurring species pair $\mathcal{N} \mathcal{P}_{t}$, identified through the cycle generation procedure. If the individual species in a pair participate in the same reactions, they form a pseudo-species since all the fast reaction rate terms cancel out. If the species individually participate in other fast reactions, they are then inserted into a SpeciesQueue, $S Q$ for identifying other cycles of these species. A species $S_{j}$ is selected from the $S Q$ and its presence in other cycles is checked. If a reaction edge that has not been processed is found, then the other species in the corresponding pair is identified and inserted in the $S Q$. If the reaction edge has already been processed, a check regarding participation of the products of the reaction edge in a different unimolecular reaction is performed. If such a reaction exists, the coefficient of species $S_{j}$ is updated within the pseudo-species generated, to account for the reaction rate terms from both the product species. A species is removed from the $S Q$ if all the reactions that the species participates in have been accounted for. The procedure ends when $n_{f}-m_{f}$ number of pseudo-species are generated, where $n_{f}$ denotes the number of species that participate in the $m_{f}$ fast/equilibrated reactions. Note that for a sub-graph with only unimolecular reactions, only one pseudo-species is generated following the above described procedure, which is the summation of all the species within the sub-graph.
\end{abstract}




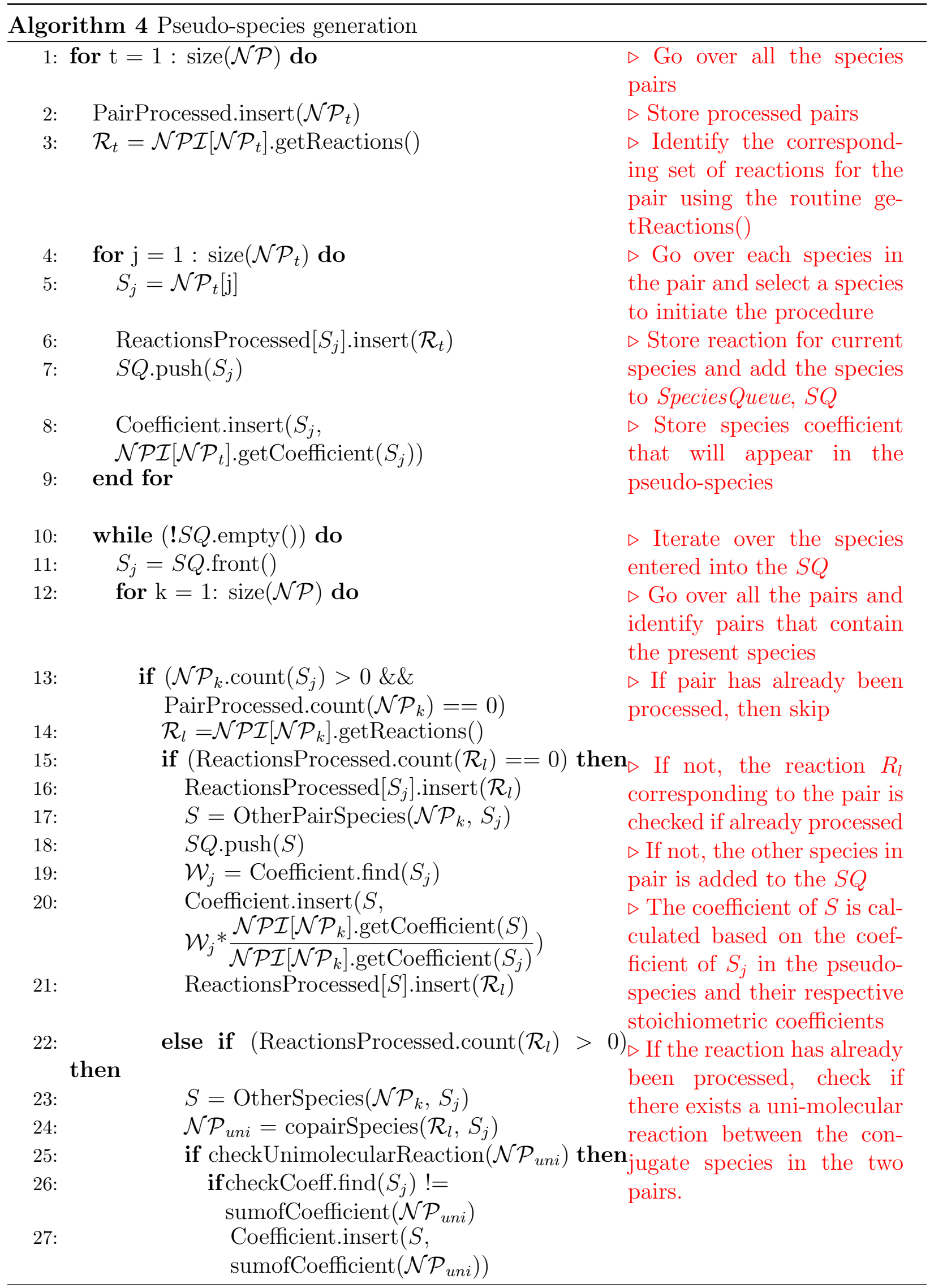




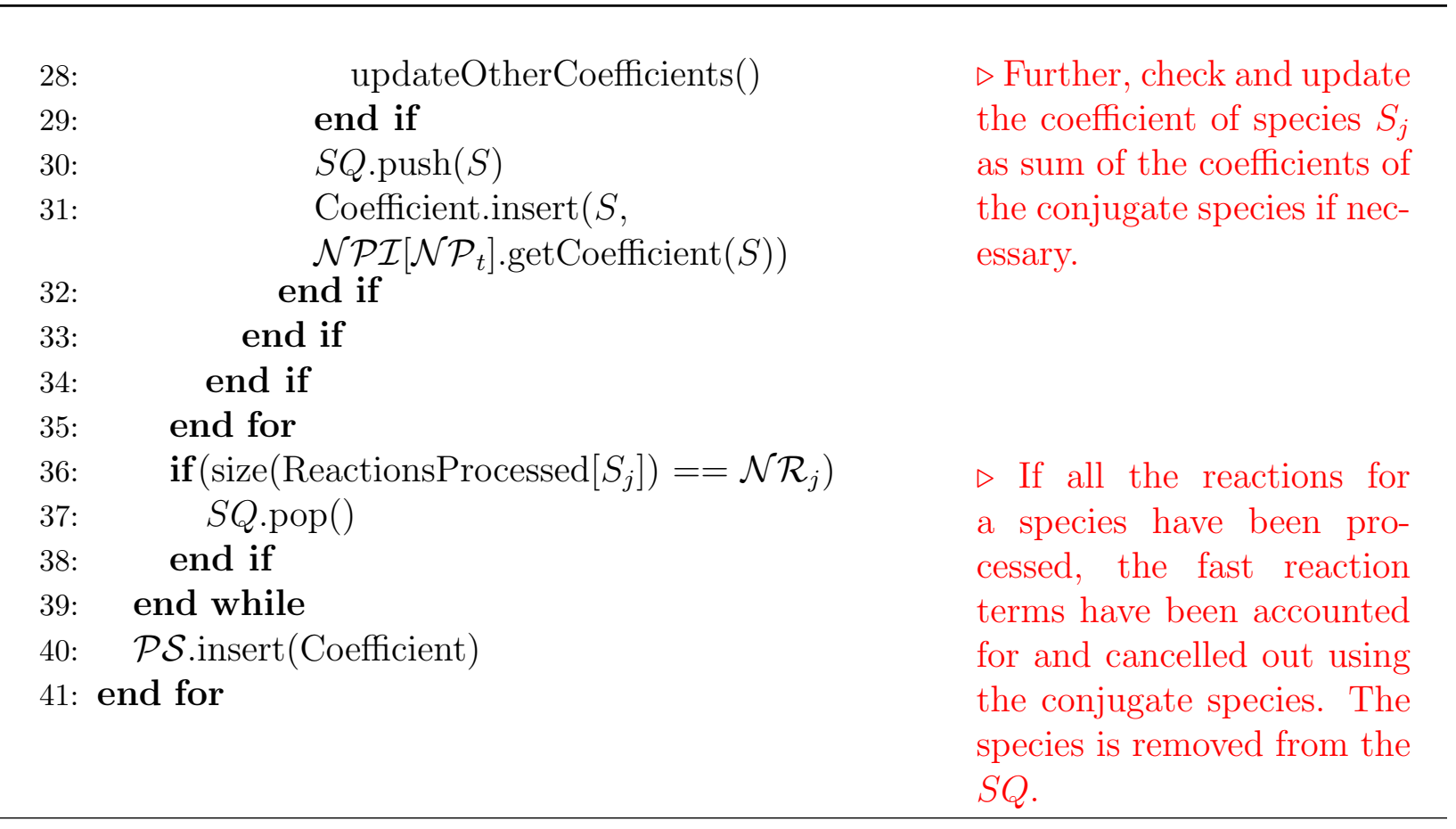

The generation of pseudo-species via the cycle identification procedure is automated in an algorithm for each sub-graph. The algorithms presented in this work are implemented as a computational tool written in $\mathrm{C}++$ to automate this graph theoretic framework; this tool is used in all the examples presented below.

\subsection{Equation formulation for the reduced model}

The fast/equilibrated reactions, identified in section 1, are used to formulate the algebraic constraints in the reduced model. Complete conversion constraints are enforced for all the fast irreversible reactions:

$$
f_{i}(\mathrm{C})=0
$$

The quasi-equilibrium assumption is enforced for all the reversible reactions:

$$
f_{i}(\mathrm{C})-\frac{k_{-i}}{k_{i}} \times f_{-i}(\mathrm{C})=0
$$

In a system involving $n_{f}$ species participating in $m_{f}$ fast reactions, Eq. 15 and Eq. 16 constitute $m_{f}$ algebraic constraints, $\mathrm{F}(\mathrm{C})=0$. The $m_{f}$ algebraic constraints are assumed independent such that the Jacobian $(\partial \mathrm{F}(\mathrm{C}) / \partial C$ ) has full row rank. If not, a subset of independent constraints are selected (Kumar et al., 1998). A reduced order description of the system (Eq. 3 or Eq. 4) in terms of the slow pseudo-species $\zeta$ of order equal to the degrees of freedom $\left(n_{f}-m_{f}\right)$, can be obtained by considering a coordinate change of the form:

$$
\left[\begin{array}{l}
\zeta \\
\eta
\end{array}\right]=T(\mathrm{C})=\left[\begin{array}{l}
\phi \times \mathrm{C} \\
F(\mathrm{C})
\end{array}\right]
$$

where $\phi$ is the coefficient matrix obtained from the generation of the pseudo-species. This essentially projects the description of the system on the equilibrium state space where $\eta=$ 
0 . The differential equations for $\zeta$ will only depend on the slow reaction rates and will take the form (referring to Eq. 3):

$$
\frac{d \zeta}{d t}=\phi \times\left.\frac{d C}{d t}\right|_{C=T^{-1}(\zeta, 0)}
$$

with initial conditions consistent with the algebraic constraints $\mathrm{F}(\mathrm{C})=0$.

Alternatively, the slow dynamics of the system can be simulated in terms of a reduced set of the original species. For this, the set of original species is partitioned into a reduced $\left(n_{f}-m_{f}\right)$ species set $C_{r}$ and the remaining ones, $\widehat{C_{r}}$. Given the full row rank of $\partial \mathrm{F}(\mathrm{C}) / \partial C$, the algebraic constraints $\mathrm{F}(\mathrm{C})=0$ can be solved for $\widehat{C_{r}}$ :

$$
\widehat{C_{r}}=F^{\prime}\left(C_{r}\right)
$$

Using Eq. 18 and Eq. 19, the slow dynamics of the system is given by

$$
\frac{d \zeta}{d t}=\phi \times \frac{d}{d t}\left[\begin{array}{c}
C_{r} \\
F^{\prime}\left(C_{r}\right)
\end{array}\right]=\phi \times\left[\begin{array}{c}
I_{n_{f}-m_{f}} \\
\frac{\partial F\left(C_{r}\right)}{\partial C_{r}}
\end{array}\right] \frac{d C_{r}}{d t}=P \frac{d C_{r}}{d t}
$$

Based on the independence assumption, it can be shown that $P$ is non-singular (Lee and Othmer, 2010) which gives

$$
\frac{d C_{r}}{d t}=P^{-1} \frac{d \zeta}{d t}
$$

The explicit representation of the reduced model is given by Eq. 21 with the initial condition for species $C_{r}$ selected so that $\mathrm{F}\left(C_{r}(0), \mathrm{F}^{\prime}\left(C_{r}(0)\right)=0\right.$.

\section{RESULTS AND DISCUSSION}

The developed graph-theoretic framework is applied to two case studies. The first case study is a reaction system involving 1-butene cracking which comprises of reversible unimolecular reactions only. In the second case study, a biochemical reaction system involving carbon metabolism in erythrocytes is considered with both reversible and irreversible bimolecular reactions.

\subsection{Cracking and isomerization of 1-butene}

1-butene cracks to form ethene and isomerizes to form 2-butene and isobutene (Chen et al., 2014). The reaction scheme containing 10 species and 15 reactions on a Bronsted-acid catalyst is shown in Figure 6. 


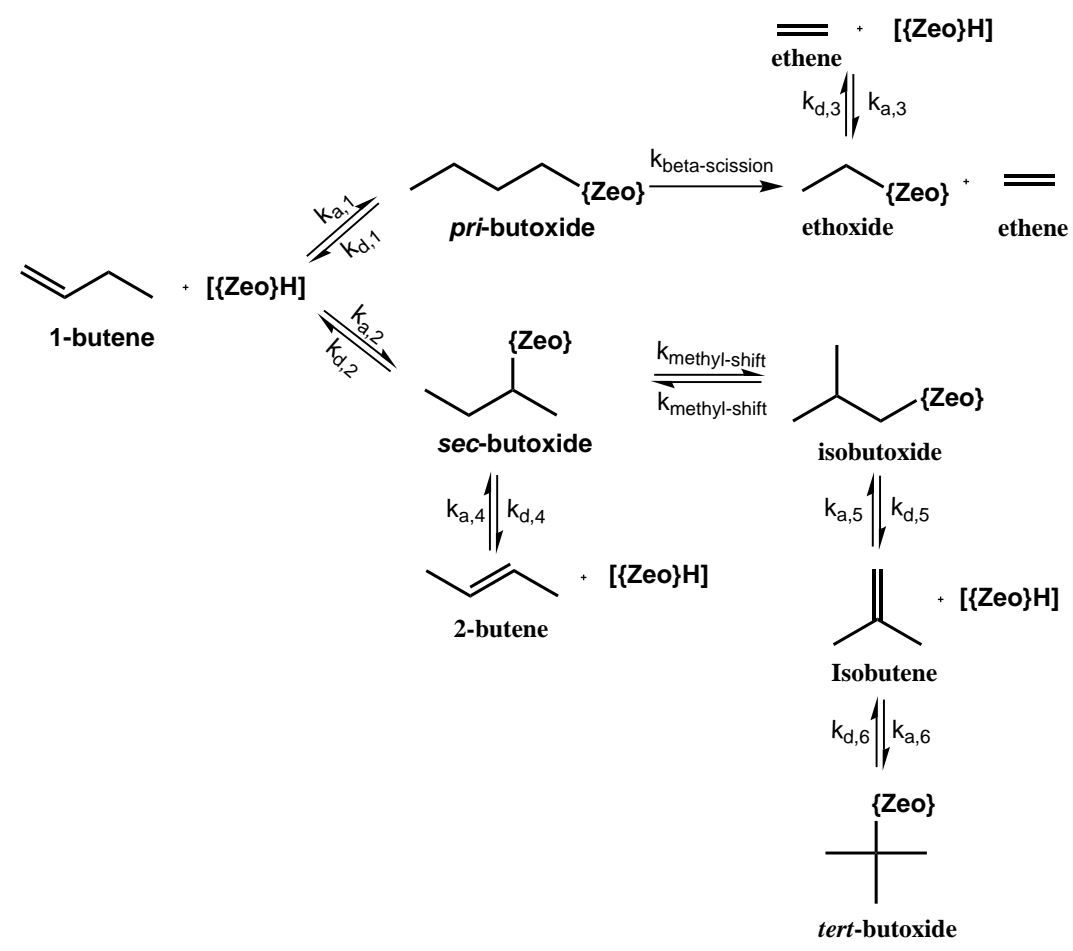

Figure 6: Reaction scheme for 1-butene cracking and isomerization; rate constants $k_{\mathrm{a}}$ correspond to the olefin adsorption reactions, $k_{\mathrm{d}}$ correspond to the desorption reactions, $k_{\text {methyl-shift }}$ correspond to the methyl shift reactions, and $k_{\text {beta-scission }}$ corresponds to the $\beta$-scission reaction. $[\{\mathrm{Zeo}\} \mathrm{H}]$ denotes the Bronsted-acid sites in a zeolite catalyst and the species containing $[\{Z e o\}]$ denote a surface alkoxide intermediate.

The kinetic parameters involving pre-exponential factors and activation energies are taken from literature reports (Chen et al., 2014; Nguyen et al., 2011, 2012) and are listed in section S.1 of the supporting information. The original model (Eq. 4) is simulated with the initial flow rate of 1-butene $=2.14 \mathrm{mmol} / \mathrm{h}$ and initial free site concentration $=0.219 \mathrm{mmol} / \mathrm{g}_{\text {cat }}$ for a temperature $\mathrm{T}=623 \mathrm{~K}$. An equilibrium tolerance of $\delta=0.05$ was used to identify fast equilibrated reversible reactions. Using this tolerance, five unimolecular reactions are found to be equilibrated and the two corresponding sub-networks are shown in Figure 7. 


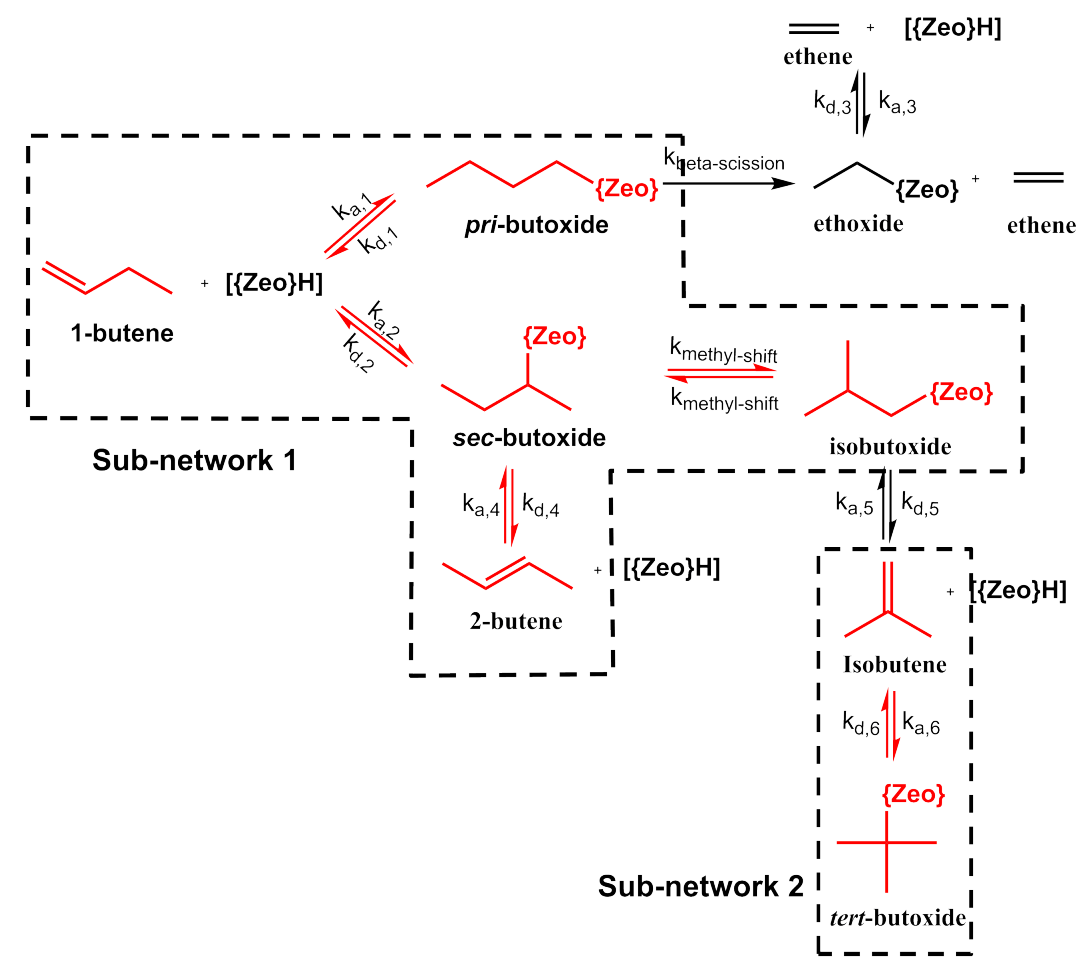

Figure 7: Fast reaction sub-networks identified for 1-butene cracking and isomerization reactions The corresponding pseudo-species generated for each sub-network are shown below:

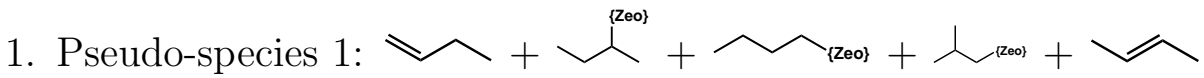

2. Pseudo-species 2: $\varkappa_{+}^{\text {\{zeo\} }}$

The reduced model is formulated using the above pseudo-species along with the initial conditions derived from the quasi-equilibrium constraints. A comparison between the original and the reduced model evolution profiles is shown in Figure 8. 

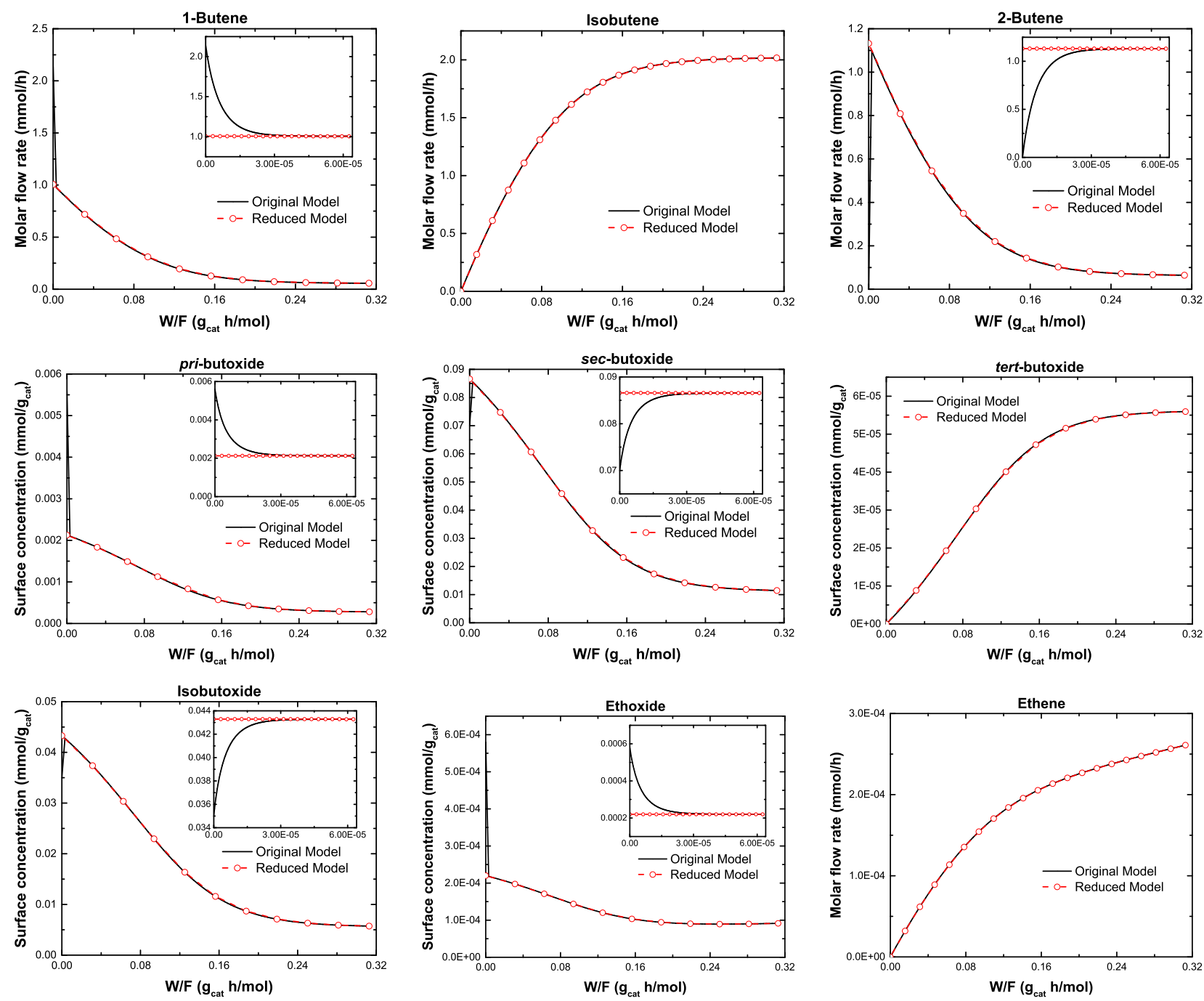

Figure 8: A comparison between the original and the reduced model evolution profiles as a function of reactor volume of various species in 1-butene cracking and isomerization reaction scheme. The solid line (-) denotes the original model and the dashed line (- $\bigcirc$-) denotes the reduced model.

The reduced model eliminates the initial fast transient due to incorporation of the quasiequilibrium constraints as shown in the insets of Fig. 8. As shown in Table 1, a significant reduction in the number of integration steps (by an order of magnitude) is observed and five (out of eight) model parameters are eliminated by employing the quasi-equilibrium approximation. 
Table 1: A comparison of integration steps and model parameters between the original and reduced models proposed for 1-butene cracking. The IDAS package (Hindmarsh et al., 2005) was used to simulate the models using a relative tolerance of $10^{-4}$ and an absolute tolerance of $10^{-6}$.

\begin{tabular}{|c|c|c|}
\hline & Original Model & Reduced Model \\
\hline No. of steps & 228 & 38 \\
\hline No. of residual evaluations & 4875 & 240 \\
\hline No. of Jacobian evaluations & 342 & 14 \\
\hline No. of non-linear iterations & 426 & 55 \\
\hline No. of model parameters & 8 & 3 \\
\hline
\end{tabular}

\subsection{Carbon metabolism in Erythrocytes}

Erythrocytes are simple systems, due to the lack of compartmentalization, allowing for the study of glycolysis with minimum interference from other pathways (see (Heinrich et al., 1978)). The reaction scheme for carbon metabolism in erythrocytes containing 20 species and 25 reactions (15 reversible and 10 irreversible) is shown in Fig. 9 (Gerdtzen et al., 2004).

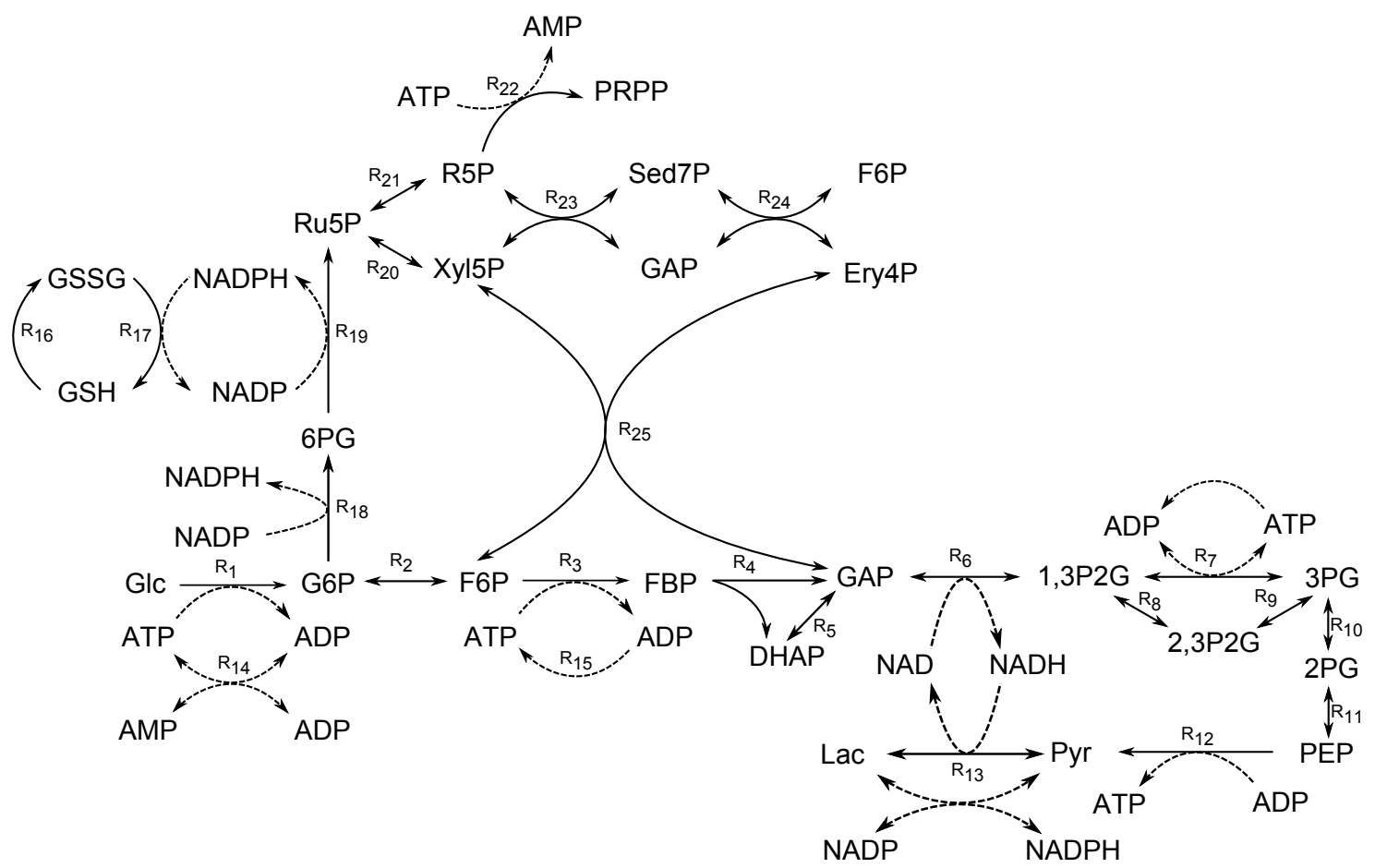

Figure 9: Reaction scheme for carbon metabolism in erythrocytes. Adapted from (Gerdtzen et al., 2004).

The rate expressions and the pseudo-first order rate constants for the system are taken from literature reports (Heinrich et al., 1978; Gerdtzen, 2005) and are listed in section S.2.1 of the supporting information. The effect of the equilibrium tolerance and kinetic threshold criteria on the accuracy and computational effort of the resulting reduced models was examined. Four different cases shown in Table 2 were considered. In case 1, only fast reversible reactions were identified by using an equilibrium index of $\delta=0.01$. The forward and reverse reaction rates were calculated through a forward simulation of the ODEs listed in the supporting 
information. In case 2 , the equilibrium index criterion was relaxed $(\delta=0.2)$ to include more fast reversible reactions. In case 3 , fast irreversible reactions were also introduced with a kinetic threshold $k^{\text {min }}=10^{6} h^{-1}$. In case 4 , the kinetic threshold was further relaxed $\left(k^{\text {min }}\right.$ $\left.=10^{5} h^{-1}\right)$. Table 2 shows the reactions selected for each case. The initial conditions for the original model are listed in Table 3. The corresponding sub-networks identified for each case are shown in section S.2.2 of the supporting information.

Table 2: Different cases considered for identifying fast/equilibrated reactions

\begin{tabular}{|c|c|c|}
\hline & Identification Criteria & Reactions assumed fast \\
\hline Case 1 & $\delta=0.01$ & $R_{5}, R_{10}, R_{11}, R_{20}$ \\
\hline Case 2 & $\delta=0.2$ & $R_{2}, R_{5}, R_{10}, R_{11}, R_{20}, R_{21}$ \\
\hline Case 3 & $\delta=0.2$ and $k^{\text {min }}=10^{6} h^{-1}$ & $R_{2}, R_{3}, R_{5}, R_{10}, R_{11}, R_{20}, R_{21}$ \\
\hline Case 4 & $\delta=0.2$ and $k^{\text {min }}=10^{5} h^{-1}$ & $R_{2}, R_{3}, R_{5}, R_{10}, R_{11}, R_{20}, R_{21}, R_{22}$ \\
\hline
\end{tabular}

Table 3: Initial conditions for erythrocytes model

\begin{tabular}{cc}
\hline$C_{\mathrm{G} 6 \mathrm{P}}=0.0385 \mathrm{mM}$ & $C_{6 \mathrm{PG}}=0.0049 \mathrm{mM}$ \\
$C_{\mathrm{F} 6 \mathrm{P}}=0.0157 \mathrm{mM}$ & $C_{\mathrm{Ru} 5 \mathrm{P}}=0.016 \mathrm{mM}$ \\
$C_{\mathrm{FBP}}=0.007 \mathrm{mM}$ & $C_{\mathrm{Xy} 15 \mathrm{P}}=0.016 \mathrm{mM}$ \\
$C_{\mathrm{GAP}}=0.0057 \mathrm{mM}$ & $C_{\mathrm{R} 5 \mathrm{P}}=0.018 \mathrm{mM}$ \\
$C_{\mathrm{DHAP}}=0.14 \mathrm{mM}$ & $C_{\mathrm{SH} 7 \mathrm{P}}=0.0199 \mathrm{mM}$ \\
$C_{1,3 \mathrm{P} 2 \mathrm{G}}=0.0005 \mathrm{mM}$ & $C_{\mathrm{E} 4 \mathrm{P}}=0.0076 \mathrm{mM}$ \\
$C_{3 \mathrm{PG}}=0.0685 \mathrm{mM}$ & $C_{\mathrm{NADP}}=0.0014 \mathrm{mM}$ \\
$C_{2,3 \mathrm{P} 2 \mathrm{G}}=5.7 \mathrm{mM}$ & $C_{\mathrm{ATP}}=1.83 \mathrm{mM}$ \\
$C_{2 \mathrm{PG}}=0.01 \mathrm{mM}$ & $C_{\mathrm{AMP}}=0.037 \mathrm{mM}$ \\
$C_{\mathrm{PEP}}=0.017 \mathrm{mM}$ & $C_{\mathrm{GSH}}=3.15 \mathrm{mM}$ \\
\hline
\end{tabular}

\subsubsection{Case 1: $\delta=0.01$}

Reactions $R_{5}, R_{10}, R_{11}$, and $R_{20}$ are treated as fast, with 7 species participating in these fast reactions. The reduced model is initialized so that the corresponding quasi-equilibrium constraints, listed in Table 4 , are satisfied at initial time $t=0$. The reduced model eliminates the initial fast transient due to the incorporation of the quasi-equilibrium constraints as shown in the insets of Figure 10.

\subsubsection{Case 2: $\delta=0.2$}

Reactions $R_{2}$ and $R_{21}$ along with reactions in case 1 are added to the set of fast reactions. The same effect in the initial concentrations and the evolution profile of the species (shown in Figure 10) in the reduced model is seen as in case 1.

\subsubsection{Case 3: $\delta=0.2$ and $k^{\min }=10^{6} h^{-1}$}

Reaction $R_{3}$ is added to the list of fast reactions and the corresponding constraint $\left(C_{\mathrm{F} 6 \mathrm{P}} C_{\mathrm{MgATP}}\right.$ $=0$ ) results in complete conversion of the reactant species F6P as seen in Figure 10. The reduced model is initialized with zero concentration of species F6P. This results in an increase in the concentration of FBP, GAP, and DHAP with time. The evolution profile of the species subsequently aligns with that of the original model. 


\subsubsection{Case 4: $\delta=0.2$ and $k^{\text {min }}=10^{5} h^{-1}$}

Reaction $R_{22}$ is added to the list of fast reactions and the corresponding constraint $\left(C_{\mathrm{R} 5 \mathrm{P}} C_{\mathrm{MgATP}}\right.$ $=0)$ results in complete conversion of the reactant species R5P as seen in Figure 10. The reduced model is initialized with zero concentration of species R5P. The equilibrium constraint imposed on reactions $R_{20}$ and $R_{21}$ result in zero initial concentration of species Xyl5P and Ru5P. Because of this, accumulation of species E4P takes place with time as can be seen in Figure 10, with the evolution profile deviating from that of the original model.

Table 4: List of the pseudo-species generated and the algebraic constraints enforced for the fast/equilibrated reactions in the different cases considered for identifying fast/equilibrated reactions

\begin{tabular}{|c|c|c|}
\hline Case & Pseudo-species generated & Algebraic constraints \\
\hline 1 & $\begin{array}{c}3 \mathrm{PG}+2 \mathrm{PG}+\mathrm{PEP} \\
\mathrm{GAP}+\mathrm{DHAP} \\
\mathrm{Ru} 5 \mathrm{P}+\mathrm{Xyl} 5 \mathrm{P}\end{array}$ & $\begin{array}{c}K_{5} \times C_{\mathrm{DHAP}}-C_{\mathrm{GAP}}=0 \\
K_{10} \times C_{3 \mathrm{PG}}-C_{2 \mathrm{PG}}=0 \\
K_{11} \times C_{2 \mathrm{PG}}-C_{\mathrm{PEP}}=0 \\
K_{20} \times C_{\mathrm{Ru} 5 \mathrm{P}}-C_{\mathrm{Xyl5P}}=0\end{array}$ \\
\hline 2 & $\begin{array}{c}3 \mathrm{PG}+2 \mathrm{PG}+\mathrm{PEP} \\
\mathrm{GAP}+\mathrm{DHAP} \\
\mathrm{R} 5 \mathrm{P}+\mathrm{Ru} 5 \mathrm{P}+\mathrm{Xyl} 5 \mathrm{P} \\
\mathrm{G} 6 \mathrm{P}+\mathrm{F} 6 \mathrm{P}\end{array}$ & $\begin{array}{c}K_{2} \times C_{\mathrm{G} 6 \mathrm{P}}-C_{\mathrm{F} 6 \mathrm{P}}=0 \\
K_{5} \times C_{\mathrm{DHAP}}-C_{\mathrm{GAP}}=0 \\
K_{10} \times C_{3 \mathrm{PG}}-C_{2 \mathrm{PG}}=0 \\
K_{11} \times C_{2 \mathrm{PG}}-C_{\mathrm{PEP}}=0 \\
K_{20} \times C_{\mathrm{Ru} 5 \mathrm{P}}-C_{\mathrm{Xyl5P}}=0 \\
K_{21} \times C_{\mathrm{Ru} 5 \mathrm{P}}-C_{\mathrm{R} 5 \mathrm{P}}=0\end{array}$ \\
\hline 3 & $\begin{array}{c}3 \mathrm{PG}+2 \mathrm{PG}+\mathrm{PEP} \\
\mathrm{GAP}+\mathrm{DHAP} \\
\mathrm{R} 5 \mathrm{P}+\mathrm{Ru} 5 \mathrm{P}+\mathrm{Xyl} 5 \mathrm{P} \\
\mathrm{G} 6 \mathrm{P}+\mathrm{F} 6 \mathrm{P}+\mathrm{FBP} \\
\mathrm{ATP}+\mathrm{FBP}\end{array}$ & $\begin{array}{c}K_{2} \times C_{\mathrm{G} 6 \mathrm{P}}-C_{\mathrm{F} 6 \mathrm{P}}=0 \\
C_{\mathrm{F} 6 \mathrm{P}} C_{\mathrm{MgATP}}=0 \\
K_{5} \times C_{\mathrm{DHAP}}-C_{\mathrm{GAP}}=0 \\
K_{10} \times C_{3 \mathrm{PG}}-C_{2 \mathrm{PG}}=0 \\
K_{11} \times C_{2 \mathrm{PG}}-C_{\mathrm{PEP}}=0 \\
K_{20} \times C_{\mathrm{Ru} 5 \mathrm{P}}-C_{\mathrm{Xyl5P}}=0 \\
K_{21} \times C_{\mathrm{Ru} 5 \mathrm{P}}-C_{\mathrm{R} 5 \mathrm{P}}=0\end{array}$ \\
\hline 4 & $\begin{array}{c}3 \mathrm{PG}+2 \mathrm{PG}+\mathrm{PEP} \\
\mathrm{GAP}+\mathrm{DHAP} \\
\mathrm{R} 5 \mathrm{P}+\mathrm{Ru} 5 \mathrm{P}+\mathrm{Xyl} 5 \mathrm{P}+\mathrm{AMP} \\
\mathrm{G} 6 \mathrm{P}+\mathrm{F} 6 \mathrm{P}+\mathrm{FBP} \\
\mathrm{ATP}+\mathrm{FBP}+\mathrm{AMP}\end{array}$ & $\begin{array}{c}K_{2} \times C_{\mathrm{G} 6 \mathrm{P}}-C_{\mathrm{F} 6 \mathrm{P}}=0 \\
C_{\mathrm{F} 6 \mathrm{P}} C_{\mathrm{MgATP}}=0 \\
K_{5} \times C_{\mathrm{DHAP}}-C_{\mathrm{GAP}}=0 \\
K_{10} \times C_{3 \mathrm{PG}}-C_{2 \mathrm{PG}}=0 \\
K_{11} \times C_{2 \mathrm{PG}}-C_{\mathrm{PEP}}=0 \\
K_{20} \times C_{\mathrm{Ru} 5 \mathrm{P}}-C_{\mathrm{Xyl} 5 \mathrm{P}}=0 \\
K_{21} \times C_{\mathrm{Ru} 5 \mathrm{P}}-C_{\mathrm{R} 5 \mathrm{P}}=0 \\
C_{\mathrm{R} 5 \mathrm{P}} C_{\mathrm{MgATP}}=0\end{array}$ \\
\hline
\end{tabular}



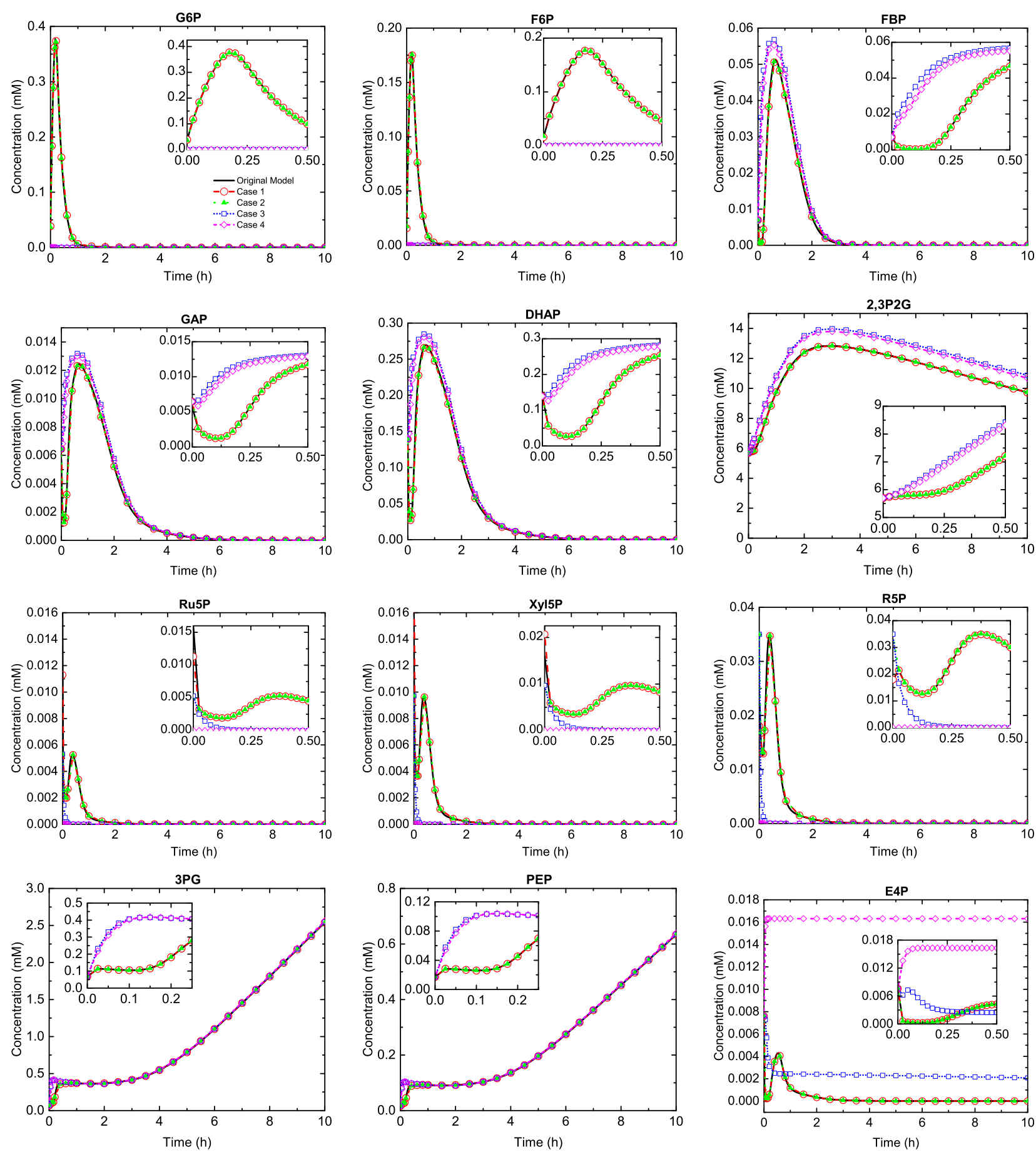

Figure 10: A comparison between original and reduced model evolution profiles of various species for the different cases of the identification criteria for fast/equilibrated reactions. The solid line (-) denotes the original model evolution profiles, the symbol (-○-) denotes the reduced model evolution profiles of various species for case 1 , the symbol $(-\triangle-)$ denotes the reduced model evolution profiles of various species for case 2 , the symbol (- $\square-$ ) denotes the reduced model evolution profiles of various species for case 3 and the symbol $(-\diamond-)$ denotes the reduced model evolution profiles of various species for case 4.

The evolution profiles of the species in Figure 10 illustrate that as more and more reactions 
are considered fast, the dynamics of the reaction system is constrained to a lower dimension. As shown in Table 5, a reduction in the integration steps is observed as additional reactions are treated as equilibrated/instantaneous. A similar decrease in the number of model parameters is observed with an increase in the number of algebraic constraints added to the reduced model.

Table 5: A comparison between the original and reduced model for carbon metabolism in erythrocytes for the different cases considered for identifying fast/equilibrated reactions listed in Table 2

\begin{tabular}{|c|c|c|c|c|c|}
\hline & $\begin{array}{c}\text { Original } \\
\text { Model }\end{array}$ & Case 1 & Case 2 & Case 3 & Case 4 \\
\hline No. of integration steps & 461 & 404 & 386 & 268 & 260 \\
\hline No. of residual evaluations & 1401 & 1354 & 1388 & 1091 & 1077 \\
\hline No. of Jacobian evaluations & 35 & 35 & 37 & 33 & 34 \\
\hline No. of non-linear iterations & 701 & 651 & 645 & 428 & 394 \\
\hline No. of model parameters & 134 & 124 & 116 & 110 & 108 \\
\hline
\end{tabular}

The results show that the proposed reduction framework allows the user to systematically address the trade-offs between accuracy and computational complexity in the resulting reduced models.

\section{CONCLUSION}

A graph-theoretic framework is developed to generate non-stiff non-linear reduced models. Within this framework, a set of pseudo-species that evolve only in the slow time scale are generated as a linear combination of original species via a cycle identification procedure. A reduced model is formulated using these pseudo-species and algebraic constraints arising from fast/equilibrated reactions. The incorporation of complete conversion or quasi-equilibrium constraints allows a reduction in the number of model parameters. The efficacy of the developed framework is illustrated through application on two chemical systems. The cracking reaction scheme of 1-butene over zeolite acids was studied and an order of magnitude reduction in the number of integration steps was observed by incorporating quasi-equilibrium constraints. Further, the trade-off between the accuracy and the computational complexity of the resulting reduced models for carbon metabolism in erythrocytes system was studied by gradually relaxing the criteria for identifying fast/equilibrated reactions. The developed graph-theoretic framework is an automatic, generic procedure that generates non-stiff reduced models of isothermal reaction systems.

\section{NOMENCLATURE}

The following symbols are used in the algorithms presented.

- $\mathcal{G}_{\mathcal{B}}(S, R, E)$ : The bi-partite graph

- $C_{0}$ : The set of initial concentration values of species

- $k$ : The set of kinetic parameters 
- IRS : The set of irreversible reactions

- $\mathcal{R S}$ : The set of reversible reactions

- $\mathcal{N}_{R}$ : The set of reactants of a reaction

- $\mathcal{N}_{P}$ : The set of products of a reaction

- $R M_{i}$ : Reactant map for species $S_{i}$ where species is a reactant

- $P M_{i}$ : Product map for species $S_{i}$ where species is a product

- $E_{\text {fast }}$ : The set of fast edges in the bi-partite graph

- $E_{\text {slow }}$ : The set of slow edges in the bi-partite graph

- $S Q$ : Queue to process the species

- $N Q$ : Queue to process the nodes while generating cycles

- $\mathcal{S G}$ : Sub-graph consisting of fast reactions only

- $\mathcal{S G S}$ : The set of species in the respective sub-graph

- $\mathcal{S G R}$ : The set of reactions in the respective sub-graph

- $\mathcal{T S}$ : The set of true slow species

- $\mathcal{N R}_{i}=\mathcal{R M}_{i}+\mathcal{P} \mathcal{M}_{i}$ : The set of reactions for species $S_{i}$

- $\mathcal{N} \mathcal{P}$ : The set of all species pairs

- $\mathcal{N P I}$ : The set of species pairs with the corresponding reaction and weights

- $w_{j}$ : Stoichiometric coefficient of corresponding species in reaction $R_{j}$

- $\mathcal{W}_{i}$ : Coefficient of corresponding species $S_{i}$ in the pseudo-species

- $\mathcal{N} \mathcal{P}_{i}$ : The set of species for a species pair

- $\mathcal{N} \mathcal{P}_{\text {uni }}$ : The set of species being checked for a uni-molecular reaction

- $\mathcal{P S}$ : The set of pseudo-species generated for a sub-graph

\section{ACKNOWLEDGEMENTS}

We acknowledge the National Science Foundation, Energy for sustainability, CDS\&E (Grant no. 1307089) for the financial support. We would also like to acknowledge Dr. Srinivas Rangarajan for insightful discussions.

\section{REFERENCES}

M. S. Okino and M. L. Mavrovouniotis. Simplification of mathematical models of chemical reaction systems. Chemical Reviews, 98(2):391-408, 1998.

S. H. Lam and D. A. Goussis. Understanding complex chemical kinetics with computational singular perturbation. Proc. Combus. Inst., 22(1):931-941, 1989.

S. H. Lam and D. A. Goussis. The CSP method for simplifying kinetics. International Journal of Chemical Kinetics, 26(4):461-486, 1994. 
A. Massias, D. Diamantis, E. Mastorakos, and D. A. Goussis. An algorithm for the construction of global reduced mechanisms with CSP data. Combustion and Flame, 117(4): 685-708, 1999.

N. Fenichel. Geometric singular perturbation theory for ordinary differential equations. Journal of Differential Equations, 31(1):53-98, 1979.

U. Maas and S. B. Pope. Simplifying chemical kinetics: Intrinsic low-dimensional manifolds in composition space. Combustion and Flame, 88(3-4):239-264, 1992.

S. B. Pope. Computationally efficient implementation of combustion chemistry using in situ adaptive tabulation. Combustion Theory and Modelling, 1(1):41-63, 1997.

B. Yang and S. B. Pope. Treating chemistry in combustion with detailed mechanismsIn situ adaptive tabulation in principal directionsPremixed combustion. Combustion and Flame, 112(1-2):85-112, 1998.

S. J. Fraser. The steady state and equilibrium approximations: A geometrical picture. The Journal of Chemical Physics, 88(8):4732-4738, 1988.

M. R. Roussel and S. J Fraser. Accurate steady-state approximations: implications for kinetics experiments and mechanism. The Journal of Physical Chemistry, 95(22):87628770, 1991.

N. Vora and P. Daoutidis. Nonlinear model reduction of chemical reaction systems. AIChE Journal, 47(10):2320-2332, 2001.

Z. P. Gerdtzen, P. Daoutidis, and W. S. Hu. Non-linear reduction for kinetic models of metabolic reaction networks. Metabolic Engineering, 6:140-154, 2004.

R. A. Adomaitis. Dynamic order reduction of thin-film deposition kinetics models: A reaction factorization approach. Journal of Vacuum Science 83 Technology A: Vacuum, Surfaces, and Films, 34(1), 2016.

E. M. Remmers, C. D. Travis, and R. A. Adomaitis. Reaction factorization for the dynamic analysis of atomic layer deposition kinetics. Chemical Engineering Science, 127:374-391, 2015.

C. H. Lee and H. G. Othmer. A multi-time-scale analysis of chemical reaction networks: I. Deterministic systems. Journal of Mathematical Biology, 60(3):387-450, 2010.

T. P. Prescott and A. Papachristodoulou. Layered decomposition for the model order reduction of timescale separated biochemical reaction networks. Journal of Theoretical Biology, 356:113-122, 2014.

D. Lebiedz. Computing minimal entropy production trajectories: An approach to model reduction in chemical kinetics. The Journal of Chemical Physics, 120(15):6890, 2004.

T. Lu and C. K. Law. On the applicability of directed relation graphs to the reduction of reaction mechanisms. Combustion and Flame, 146(3):472-483, 2006. 
T. Lu, C. K. Law, C. S. Yoo, and J. H. Chen. Dynamic stiffness removal for direct numerical simulations. Combustion and Flame, 156(8):1542-1551, 2009.

P. Pepiot-Desjardins and H. Pitsch. An efficient error-propagation-based reduction method for large chemical kinetic mechanisms. Combustion and Flame, 154(1-2):67-81, 2008.

R. G. Susnow, A. M. Dean, W. H. Green, P. Peczak, and L. J. Broadbelt. Rate-Based Construction of Kinetic Models for Complex Systems. The Journal of Physical Chemistry A, 101(20):3731-3740, 1997.

J. R. Bowen, A. Acrivos, and A. K. Oppenheim. Singular perturbation refinement to quasisteady state approximation in chemical kinetics. Chemical Engineering Science, 18(3): 177-188, 1963.

M. Mincheva and M. R. Roussel. Graph-theoretic methods for the analysis of chemical and biochemical networks. I. Multistability and oscillations in ordinary differential equation models. Journal of Mathematical Biology, 55(1):61-86, 2007.

P. Holme, M. Huss, and H. Jeong. Subnetwork hierarchies of biochemical pathways. Bioinformatics, 19(4):532-538, 2003.

M. Domijan and M. Kirkilionis. Graph theory and qualitative analysis of reaction networks. Networks and Heterogeneous Media, 3(2):295-322, 2008.

R. Heinrich, S. M. Rapoport, and T. A. Rapoport. Metabolic regulation and mathematical models. Progress in Biophysics and Molecular Biology, 32:1-82, 1978.

T. H Cormen, C. E. Leiserson, and R. L. Rivest. Introduction to Algorithms, Second Edition, volume 7. The MIT Press, England, 2001.

R. Tarjan. Enumeration of the Elementary Circuits of a Directed Graph. SIAM Journal on Computing, 2(3):211-216, 1973.

A. Kumar, P. D. Christofides, and P. Daoutidis. Singular perturbation modeling of nonlinear processes with nonexplicit time-scale multiplicity. Chemical Engineering Science, 53(8): 1491-1504, 1998.

C.-J. Chen, S. Rangarajan, I. M. Hill, and A. Bhan. Kinetics and Thermochemistry of $\mathrm{C}_{4}-$ $\mathrm{C}_{6}$ Olefin Cracking on H-ZSM-5. ACS Catalysis, 4(7):2319-2327, 2014.

C. M. Nguyen, B. A. De Moor, M.-F. Reyniers, and G. B. Marin. Physisorption and Chemisorption of Linear Alkenes in Zeolites: A Combined QMPot(MP2//B3LYP:GULP)-Statistical Thermodynamics Study. The Journal of Physical Chemistry C, 115(48):23831-23847, 2011.

C. M. Nguyen, B. A. De Moor, M.-F. Reyniers, and G. B. Marin. Isobutene Protonation in H-FAU, H-MOR, H-ZSM-5, and H-ZSM-22. The Journal of Physical Chemistry C, 116 (34):18236-18249, 2012. 
A. C. Hindmarsh, P. N. Brown, K. E. Grant, S. L. Lee, R. Serban, D. E. Shumaker, and C. S. Woodward. Sundials: Suite of nonlinear and differential/algebraic equation solvers. ACM Transactions on Mathematical Software, 31(3):363-396, 2005.

Z. P. Gerdtzen. Modeling, analysis and theoretical exploration of the metabolism of mammalian cells in culture. PhD thesis, University of Minnesota, 2005. 